\title{
The Liquid Argon Jet Trigger of the H1 Experiment at HERA
}

\author{
Bob Olivier ${ }^{1}$, Ana Dubak-Behrendt ${ }^{1,2}$, Christian Kiesling $^{1}$, Burkard Reisert ${ }^{1}$, \\ Adil Aktas ${ }^{3}$, Biljana Antunovic ${ }^{1,3}$, Juraj Bracinik ${ }^{1}$, Charles Braquet ${ }^{1}$, Horst Brettel ${ }^{1}$, Barbara Dulny ${ }^{1}$, \\ Jürgen Fent ${ }^{1}$, Markus Fras ${ }^{1}$, Walter Fröchtenicht ${ }^{1}$, Werner Haberer ${ }^{1}$, Dirk Hoffmann ${ }^{4}$, Miriam Modjesch ${ }^{1}$, \\ Ringaile Placakyte ${ }^{1,3}$, Thomas Schörner-Sadenius ${ }^{3}$, Andreas Wassatsch ${ }^{1}$, Jens Zimmermann ${ }^{1}$
${ }^{1}$ Max-Planck-Institut für Physik (Werner-Heisenberg-Institut), Föhringer Ring 6, D-80805 München, Germany
${ }^{2}$ Faculty of Nat. Sci. and Math., University of Montenegro, P.O. Box 211, 81001 Podgorica, Montenegro ${ }^{a}$
${ }^{3}$ Deutsches Elektronen-Synchrotron DESY, P.O.Box D-22603 Hamburg, Germany
${ }^{4}$ CPPM, CNRS/IN2P3 - Univ. Mediterranee, 163 avenue de Luminy, 13288 Marseille Cedex 9, France

${ }^{a}$ Supported by the Deutsche Forschungsgemeinschaft

\begin{abstract}
We report on a novel trigger for the liquid argon calorimeter which was installed in the H1 Experiment at HERA. This trigger, called the "Jet Trigger", was running at level 1 and implemented a real-time cluster algorithm. Within only $800 \mathrm{~ns}$, the Jet Trigger algorithm found local energy maxima in the calorimeter, summed their immediate neighbors, sorted the resulting jets by energy, and applied topological conditions for the final level 1 trigger decision. The Jet Trigger was in operation from the year 2006 until the end of the HERA running in the summer of 2007 . With the Jet Trigger it was possible to substantially reduce the thresholds for triggering on electrons and jets, giving access to a largely extended phase space for physical observables which could not have been reached in $\mathbf{H 1}$ before. The concepts of the Jet Trigger may be an interesting upgrade option for the LHC experiments.
\end{abstract}

\section{INTRODUCTION}

One of the most successful tools for unraveling the structure of the constituents of the nucleus, the proton and the neutron, is deep-inelastic scattering (DIS), using charged leptons to probe nuclear targets. During the past decade, the experiments carried out at the electron ${ }^{1}$-proton collider HERA mark the culmination of such investigations, which started with the Nobel prize winning experiments of R. Hofstadter [1] and of R.E. Taylor at SLAC [2]. While these experiments used stationary nuclear targets, the HERA machine was the first (and so far the only) electron-proton collider in the world. With HERA, the center-of-mass energy, and thereby the spatial resolution to probe the inner structure of the nucleon, was brought to a new regime. With its proton beam energy of $920 \mathrm{GeV}$ and an electron energy of $27.6 \mathrm{GeV}$, HERA exceeded the previous

Manuscript version January 3, 2010

Corresponding authors: Christian Kiesling cmk@mpp.mpg.de, Bob Olivier olivier@mpp.mpg.de, Ana Dubak-Behrendt dubak@mpp.mpg.de, Burkard Reisert reisert@mpp.mpg.de

${ }^{1}$ Throughout this paper the term electron is used to denote both electrons and positrons, unless the charge state is specified explicitly. experiments by more than an order of magnitude in centerof-mass energy, achieving momentum transfers squared up to $10^{5} \mathrm{GeV}^{2}$ and a spatial resolution down to $10^{-18} \mathrm{~m}$, roughly one thousands of the proton diameter.

As it turned out, the exciting "new physics" at HERA is connected with the gluon structure of the proton. The corresponding measurements are done at very low values of the Bjorken variable $x$, which signifies the momentum fraction carried by the proton constituent interacting with the incoming electron. Low values of $x$, reaching down to $10^{-5}$, generally mean low energies of the particles created in the hadronization process of the struck proton constituent.

Triggering at a hadron machine like HERA is a challenge, and most importantly so, if the interesting physics signals lead to low energy depositions in the calorimeter. Using the liquid argon (LAr) technique, where the ionization charge from traversing particles is collected before the first amplification stage, poses a special problem with low level signals, being vulnerable to pickup and other electronic noise. In a typical calorimeter trigger the energy depositions in the entire calorimeter are summed and discriminated, and one therefore faces the problem of summing up noise from a (large) area where no particle energy deposition is found. Such additional noise clearly prevents the trigger thresholds from being set at very low levels.

In the Jet Trigger concept described here, we have addressed this problem by following a scheme where only those regions in the calorimeter are considered where localized energy depositions are found. Such depositions have a high chance of being generated by "physics", rather than by "noise". The challenge here was to fit a digital search algorithm into dedicated hardware, able to deliver a trigger decision within $800 \mathrm{~ns}$, starting with the point in time where the analog signals from the preamplifier electronics have been delivered to the Jet Trigger. Previous reports on the Jet Trigger, mostly presented at conferences, are given in [3]-[8]. 
The article is organized as follows: We first give a short description of HERA as well as of the $\mathrm{H} 1$ experiment and its multi-level trigger scheme. In section II we present some details on the "old" global liquid argon calorimeter trigger (referred to as "LAr Trigger" throughout this paper) and its limitations, before we turn to the description of the Jet Trigger concept in section III. In section IV we describe details of the hardware realization of the Jet Trigger, going through the various electronic entities which build up the functionality of the Jet Trigger. In section V we give a few examples of physics results obtained with the Jet Trigger. Finally, in section VI we present the conclusions.

\section{A. HERA and the HI Experiment}

The electron-proton collider HERA [9] at the Deutsches Elektronen-Synchrotron DESY in Hamburg provided up to now the highest available center-of-mass energies for the collision of electrons or positrons with protons. Due to its high center-of-mass energy of up to $320 \mathrm{GeV}$ and the extremely wide range in the kinematic variables $x$ (the Bjorken scaling variable) and $Q^{2}$ (the negative square of the four-momentum transfer between the incoming and outgoing electron), HERA offers a much deeper view into the inner structure of the proton than was possible before. HERA also gives access to sensitive tests of the Standard Model, in particular in the sector of strong interactions, described by quantum chromodynamics (QCD).

To measure the scattered electrons as well as the produced hadronic jets with high precision, the $\mathrm{H} 1$ collaboration has constructed a general purpose detector, optimized for precise measurements of leptons and jets. In its upgraded version (see below), the detector was equipped with five layers of multi-wire proportional chambers (MWPC), a set of central jet chambers (CJC) as well as drift chamber systems in the forward and backward regions, and silicon trackers over almost the full solid angle. Here, the directions of proton and electron beams define the forward and backward regions, respectively. The tracking system was surrounded by a highly granular LAr sampling calorimeter, separated in an electromagnetic and a hadronic part. The absorber materials were lead for the electromagnetic part and stainless steel for the hadronic part. The calorimeter was surrounded by a $1.2 \mathrm{~T}$ solenoid, and the flux return yoke was equipped with muon chambers. More details on the $\mathrm{H} 1$ detector can be found elsewhere [10], [11].

It should be stressed that the detector was originally designed to measure high $Q^{2}$ and high $x$ physics. When HERA started taking data in 1992, it came as a big surprise to observe a dramatic rise of the gluon distribution within the proton towards low $x$. To trigger this low $x$ physics, low energy thresholds for the scattered electrons and jets were needed. As a consequence, the electronics of the LAr Trigger was upgraded with special low-noise amplifiers (see section II).

When, after a few years of running, it became evident that the HERA luminosity would not be sufficient for a detailed study of the high $Q^{2}$ physics, an ambitious luminosity upgrade program was launched, the HERA II phase [12], [13]. The upgrade program of the HERA collider was performed in the years 2000-2002 and succeeded to increase the instantaneous luminosity by a factor of about three. As essential components, superconducting dipoles were installed in the vicinity of the interaction regions of the $\mathrm{H} 1$ and ZEUS experiments. In addition, spin rotators were installed before and after the interaction regions of the collider experiments in order to provide longitudinal polarization for the electron beams (left and right handed polarization for both charge states, $e^{+}$and $e^{-}$), giving access to sensitive tests of the electroweak theory and allowing unique searches for new heavy particles.

In the course of the HERA upgrade the collider experiments were upgraded as well [14]. For H1 this meant to build a new inner proportional chamber and add a silicon vertex detector in the forward region. Also the backward direction needed to be modified (silicon tracker and calorimeter) because of the additional superconducting magnets close to the $\mathrm{H} 1$ interaction region. Finally, substantial upgrades were implemented for several trigger systems, such as the Fast Track Trigger (FTT) based on the central jet chambers [15], [16], and the Jet Trigger for the LAr calorimeter which is described in this paper.

Due to the presence of additional machine components close to the interaction region, a significant increase of the background rates was expected and indeed observed after the HERA II upgrade. While parts of the $\mathrm{H} 1$ detector were upgraded as mentioned above, the $\mathrm{H} 1$ data logging rate to permanent storage (up to $50 \mathrm{~Hz}$ ) remained a stringent constraint for the data acquisition system. The aim of the upgrade of the digital part of the LAr Trigger, the Jet Trigger, was to complement the existing global LAr calorimeter trigger with a system which performs real-time clustering to avoid summingup noise distributed over large parts of the calorimeter. Such a scheme allowed for triggers on even lower energy depositions while keeping the trigger rates within the required bounds.

\section{B. The H1 Trigger System}

In experimental high energy physics (HEP), information on the elementary building blocks of matter and the forces between them is extracted from the debris of collisions of intense, high energy particle beams produced in giant accelerators. The extremely low signal to background ratio for the desired physics processes, typically 3 to 5 orders of magnitude for electron-proton interactions, presents major challenges for the design of data acquisition systems of HEP experiments. In experiments at the major accelerator facilities, data produced in building-sized multi-layered particle detectors surrounding the interaction regions are accumulated at rates of several gigabytes per second.

As it is not feasible, using today's technology, to log all of these data onto permanent storage media for later analysis, an online decision-making system is necessary: the experiment trigger. The timing requirements for such a device are rather severe. At the HERA facility, for example, a new frame of detector data arrived at the trigger system every $96 \mathrm{~ns}$, corresponding to the crossing frequency of the electron and proton bunches (bunch crossing frequency). For $\mathrm{H} 1$, the latency before the start of the full detector readout could not exceed $20 \mu \mathrm{s}$. Therefore, the HERA experiments were coping with timing constraints quite similar to those of the experiments 


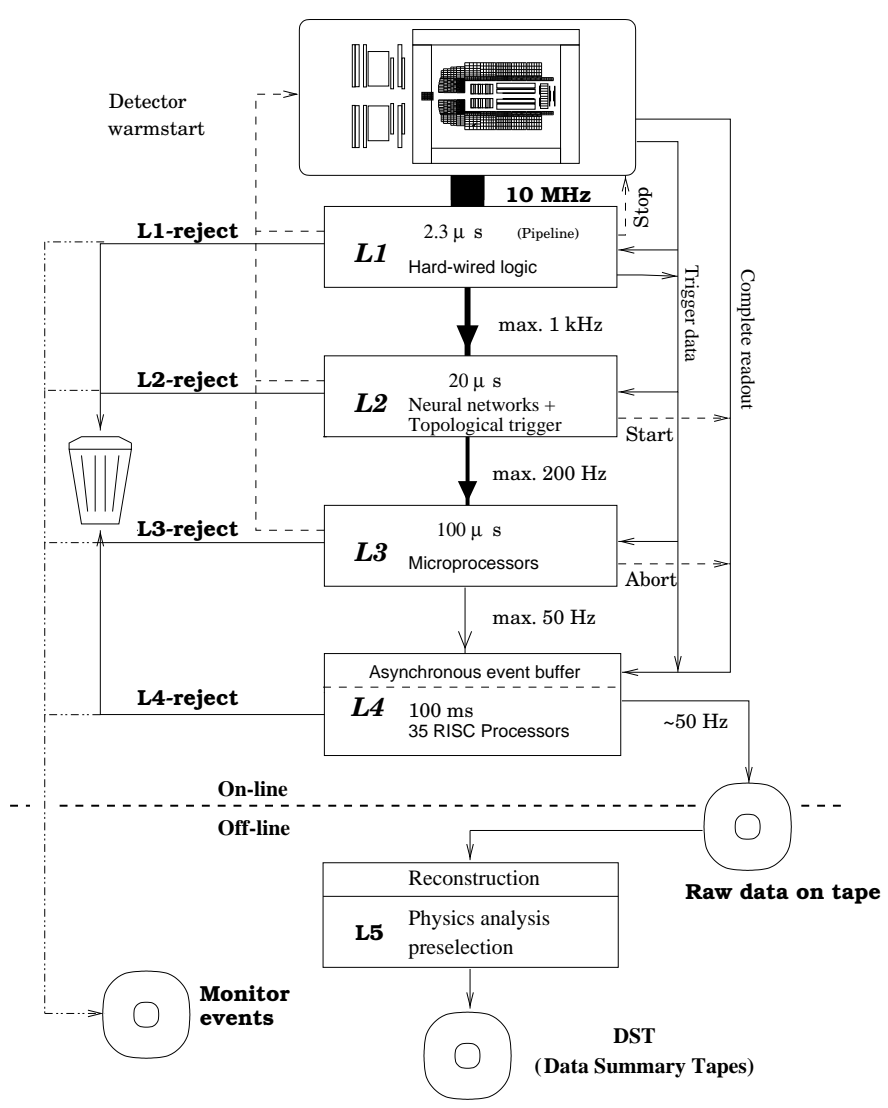

Fig. 1. Schematic view of the H1 multi-level trigger system. The input and output rates of each trigger level as well as the decision times are shown. The rate is reduced from the input value of $10.4 \mathrm{MHz}$ to an output rate of at most $50 \mathrm{~Hz}$.

at the LHC facility currently under commissioning at CERN in Switzerland, where the colliding proton bunches meet each other every $25 \mathrm{~ns}$.

Large background rates are typical for hadron machines, and may exceed, as mentioned above, by many orders of magnitude the rates expected from the physical processes of interest. To deal with this problem, H1 used a four-level trigger scheme, where the trigger rate was reduced step by step, and the decisions were improved as a result of the more complete information and more sophisticated trigger algorithms at the later trigger levels. The scheme realized in the $\mathrm{H} 1$ experiment (after the upgrade) is shown in figure 1. The first trigger level was implemented in custom-built electronics, with dedicated trigger systems for the MWPC, the drift chamber, the calorimeters, and the muon system. Additional first level triggers were realized from the forward and backward systems of the $\mathrm{H} 1$ detector. The level 1 triggers were using a pipeline technique and were therefore deadtime-free. The level 1 triggers had to decide within $2.3 \mu \mathrm{s}$. After the upgrade, the level 1 systems typically yielded a rate up to $1 \mathrm{kHz}$. Since most of this rate was still background, it had to be strongly reduced to the "physics rate" of about $10-50 \mathrm{~Hz}$, going to permanent storage. This was achieved in three further trigger levels: The second level was realized by dedicated microprocessors, a track-based trigger [15], and a neural network trigger [17], [18] with inputs from all the first level trigger systems, making a "global" event decision. A third system was dedicated mainly to reduce the trigger rate from the backward (electron direction) calorimeter [19]. The second level needed about $20 \mu \mathrm{s}$ for the trigger decision, reducing the trigger rate to about $200 \mathrm{~Hz}$. This rate was further reduced at the third trigger level which employed track-based triggers and special combinations of the FTT with the Jet Trigger, to be described later. With an "accept" by the third level the event readout was started. Finally, at the fourth level a full event reconstruction was performed on a Linux processor farm. The level 4 input rate was not exceeding $50 \mathrm{~Hz}$ by applying additional prescale factors for some triggers. A prescale factor with value $n$ meant that only every $n$-th accepted event was recorded. Further details on the H1 trigger systems can be found elsewhere [20].

In the following we concentrate on the LAr calorimeter triggers. We first present the trigger as it was designed and implemented in its pre-upgrade version, motivate the need for the upgrade to the Jet Trigger, and then turn to its principles and its realization, as well as showing examples of its physics performance.

\section{Liquid ARgOn CALORIMETER TRIgGeR}

Here, we give a description of the hardware components of the LAr Trigger, following the trigger signal from the LAr calorimeter cells up to the trigger elements which are supplied to the central trigger logic (CTL). To identify events with large momentum transfer, the LAr Trigger derives timing signals, energy signals, energy sums and trigger elements constructed from the energy sums. A schematic view of the LAr Trigger is shown in figure 2. The trigger is divided into an analog and a digital part.

\section{A. Analog Part}

The analog signal has its origin in the ionization produced by the particles in an electromagnetic (EM) or hadronic (HAD) shower. The ionization charges are generated mainly by the charged shower particles traversing the LAr gaps between the absorber materials. These charges are collected on 45000 geometric pads (GP) and the resulting signals are fed into analog cards located in the analog boxes (ANBX) mounted on the outside of the LAr cryostat. On the analog cards, the charges collected on the GPs are amplified and the signals are transformed to a bipolar (sinusoidal) shape with an amplitude proportional to the deposited energy. The width of the shaped signals fed into the trigger is about $1 \mu \mathrm{s}$. The signals of the GPs are summed up into 4845 trigger cells (TC), covering almost the entire solid angle, where one EM TC comprises 16 neighboring GPs and one HAD TC contains 4 GPs. The TC signals are then transferred to the trigger merging board (TMB) where the signals belonging to projective trigger towers (TT), pointing to the interaction region, are grouped together. The actual summing of the TCs to a TT is done in the Summing and Shaping Modules (SSM) where the TC signals can be adjusted individually in amplitude and timing. Up to 4 EM cells give one EM tower, up to 6 HAD cells give one HAD tower. In order to suppress large unphysical signals (for example from frequent high voltage discharges in a particular 


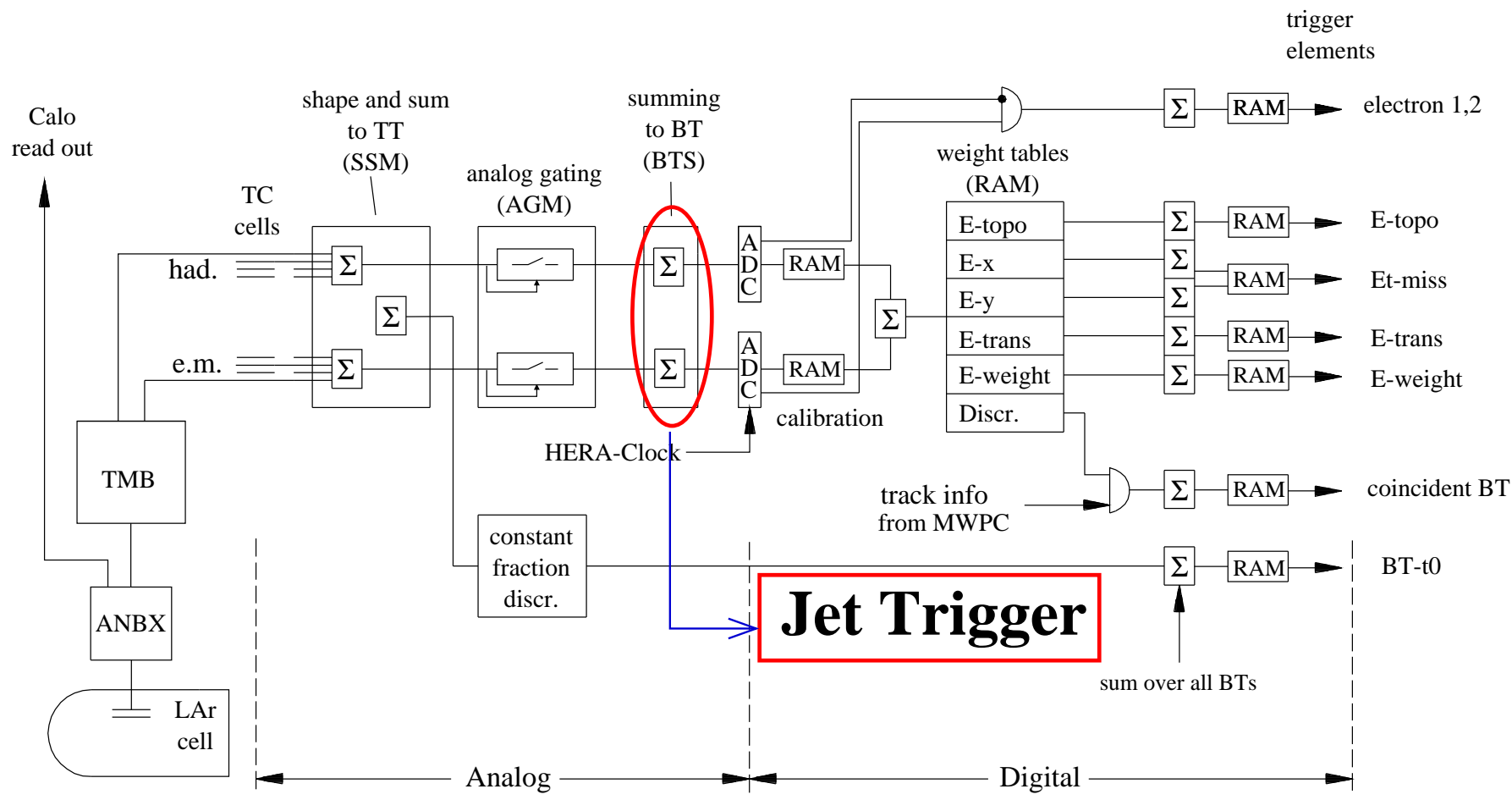

Fig. 2. Schematic view of the LAr Trigger electronics before the Jet Trigger upgrade. The LAr Trigger is divided into an analog and a digital part. The Jet Trigger receives analog signals branching off from the Big Tower Summing unit BTS.

LAr gap) the signal from each individual trigger cell can be excluded from the summation. After the SSM, the TT signals are discriminated in the Analog Gating Modules (AGM) to suppress remaining electronic noise: Both EM and HAD parts of the TT signal are compared to adjustable thresholds for each TT independently. Signals which are lower than the requested thresholds are suppressed. As the last step in the analog chain, the TTs (a total of about 1200) are summed up to big towers (BT, total of 512) in the Big Tower Summing units (BTS). The EM and HAD signals from up to four TTs are summed separately to yield the EM and HAD big tower energies which are then digitized in an 8-bit FADC system (see below).

An important aspect of the LAr Trigger is its capability to determine the time of interaction, the so called bunch crossing time or event $\mathrm{t} 0$. As the shaped signals in the trigger stretch over about 1 microsecond, a timing signal for each TT is derived by delaying the shaped pulse by $500 \mathrm{~ns}$ and comparing to the original, undelayed pulse. The crossing point of both signals is independent of the amplitude. This TT-level signal is synchronized with the HERA clock and single bunch crossings are flagged. A logical OR of all t0s belonging to a given BT defines the $\mathrm{t} 0$ of this BT. The corresponding BT-level to bits are transmitted individually to a digital adder tree where the global event t0 is calculated.

To avoid distortions of the signal by noise and to guarantee stability in tagging the correct bunch crossing, the analog t0 is formed only for those TTs which exceed a sufficiently high energy threshold of approximately $5 \mathrm{GeV}$ in the central region, rising to $10 \mathrm{GeV}$ in the forward region.

At the trigger level (TT granularity), the calorimeter is di- vided into 23 polar angle bins $(\theta)$ and up to 32 azimuthal angle bins $(\phi)$, depending on the $\theta$ position. The to thresholds are $\theta$ dependent and higher in the forward region since large energy depositions and narrow jets are expected there. In addition, high rates of t0 signals are expected in the very forward region due to remnants of the target jets and background from beamgas interactions. During the HERA operation, the three most forward $\theta$ rings were therefore excluded (masked) and did not contribute to the event $t 0$. The masking was done in the digital part of the LAr Trigger (see below).

After masking the BTs in the forward direction, all remaining t0s are counted for each bunch crossing. The total number is compared to a threshold, and a t0 trigger element is generated if a programmable threshold is exceeded. This signal is called the digital t0. At the same time the so-called analog t0 is formed by summing all t0s without masking. Again the sum is discriminated against a threshold and the resulting signal is sent to the CTL.

\section{B. Digital Part}

The digital part of the LAr Trigger receives the analog signals of the $512 \mathrm{BTs}$, half of them electromagnetic and half hadronic, as well as the to bits of the timing signals from the BTs. The digital part processes this information to provide trigger elements (TE) to the CTL. The pulses from the EM and HAD parts of the BTs are digitized in Fast Analog to Digital Converters (FADC) with a precision of 8 bits, clocked at a frequency of $10.4 \mathrm{MHz}$, corresponding to the bunch crossing frequency (HERA clock). 
In the so-called adder tree the topological sums from the FADC energies deposited in the various topological calorimeter regions (very forward (IF), forward barrel (FB) and central barrel $(\mathrm{CB})$ ), are calculated. The FADCs from the EM and HAD parts are weighted by lookup tables (denoted by RAM in figure 2) and are then added to form the energy sums quadrant by quadrant for each of the three topological regions. The weighting takes care of the proper energy calibration of each BT and compensates the remaining effects from the different energy depositions in the EM and HAD parts of the calorimeter. Before summing, the weighted BTs are discriminated against individual thresholds. The topological sums are finally compared to a set of three programmable thresholds, building up a first set of trigger elements. These bit quantities are sent to the CTL and to the trigger systems at the second trigger level.

There are also two "global" trigger elements calculated, the transverse energy sum LAr_Etrans, and the missing transverse energy LAr_Etmiss. The quantity LAr_Etrans is the scalar sum of the transverse energies of all BTs:

$$
\text { LAr_Etrans }=\sum_{\mathrm{BT}_{i}} E_{i} \sin \theta_{i}
$$

where $\theta_{i}$ is the polar angle of the big tower $\mathrm{BT}_{i}$.

The missing transverse energy sum LAr_Etmiss is the modulus of the transverse vector sum:

$$
\text { LAr_Etmiss }=\sqrt{E_{x}^{2}+E_{y}^{2}}
$$

where:

$$
\begin{aligned}
& E_{x}=\sum_{\mathrm{BT}_{i}} E_{x_{i}}=\sum_{\mathrm{BT}_{i}} E_{i} \sin \theta_{i} \cos \phi_{i} \\
& E_{y}=\sum_{\mathrm{BT}_{i}} E_{y_{i}}=\sum_{\mathrm{BT}_{i}} E_{i} \sin \theta_{i} \sin \phi_{i} .
\end{aligned}
$$

Here, $\phi_{i}$ is the azimuthal angle of the big tower $\mathrm{BT}_{i}$. All BTs in the first two $\theta$ bins of the forward region are excluded from the LAr_Etrans and LAr_Etmiss sums because in this region large energy deposition from beam-gas background is expected, leading to unacceptably high trigger rates. The functions $\sin \phi_{i}$ and $\cos \phi_{i}$ give rise to signed values for the BT energies for each component $x$ or $y$. If all particles in the final state would deposit their total energy in the calorimeter, $E_{x}$ and $E_{y}$ would be zero because of transverse momentum conservation. For example, in neutral-current events, which proceed via the exchange of a $\gamma$ or a $Z^{0}$ boson, the scattered electron is observed in the final state and, in the ideal case, perfectly balances the transverse momentum of the hadronic final state (LAr_Etmiss $\approx 0)$. The chargedcurrent processes, however, which are mediated by a $W^{ \pm}$ boson exchange, are characterized by large missing transverse energy (LAr_Etmiss $\gg 0$ ) due the final state neutrino which escapes detection.

In addition to these kinematically motivated global triggers, there is one freely programmable sum:

$$
\text { LAr_Eweight }=\sum_{\mathrm{BT}_{i}} f\left(\theta_{i}, \phi_{i}, E_{i}\right)
$$

where an arbitrary weighting function $f\left(\theta_{i}, \phi_{i}, E_{i}\right)$ can be realized, similar to $\sin \theta_{i}$ and $\cos \phi_{i}$ above, via lookup tables. Each of the adder tree sums are compared to three different thresholds, stored as discriminator outputs, and the resulting bits are sent to the CTL.

Finally, the LAr Trigger provides dedicated triggers for electrons, based on the energy depositions in the EM and HAD parts. For each BT a minimum energy is required in the EM part and a maximum energy in the HAD part. If both conditions are fulfilled, an electron bit is set for the corresponding BT. In the trigger configuration used for the HERA data taking, the hadronic veto was not used to avoid false vetos due to electronic noise and electromagnetic showers leaking into the hadronic section of the calorimeter.

The electron bits are counted and their total number is compared to a threshold. Two sets of energy thresholds are realized in the hardware, so that an "electron 1" and an "electron 2" trigger can be provided to the CTL. In addition, another bit is set for each BT with energy above $1.5 \mathrm{GeV}$ when a track segment from the MWPC is pointing to this BT. These BT bits are not only sensitive to the energy deposits of the scattered electrons but also to the localized energy deposits of jets. Therefore they were employed to trigger neutral-current as well as charged-current events (see, for example, [21], [22]).

\section{Limitations and Upgrades of the LAr Trigger}

The LAr Trigger, which was a first level trigger, had the task of providing fast identification of a hard scale in deep-inelastic scattering events, typically events with large transverse momentum $p_{T}$. Best sensitivity to physics is achieved with lowest possible energy thresholds which, however, inevitably lead to high trigger rates. The limited bandwidth of the H1 data acquisition system, on the other hand, constrained the acceptable rate of the LAr Trigger.

During the early running periods at HERA it became clear that thresholds much lower than originally planned were desirable, mainly driven by the surprising physics discovered at low $x$. Several steps toward lowering thresholds were taken, until detailed studies showed that the trigger thresholds had reached their theoretical limit, determined by the white noise in the preamplifier electronics coupled to the capacities of the calorimeter cells. To be able to decrease the thresholds even further without increasing the trigger rate, a new preamplifier hybrid using a special low-noise JFET was designed. The new low-noise preamplifiers were installed in 1998 on those analog cards which were connected to large detector capacities.

In order to fully profit from the low-noise electronics, further elements in the trigger electronics were upgraded as well. As one consequence of all these improvements, a substantial decrease in threshold was achieved [21], giving access to neutral-current reactions with high inelasticity $y$, where the contribution of the longitudinal structure function $F_{L}$ to the inclusive cross section becomes sizeable.

As the LAr Trigger employed energy summing over large parts of the calorimeter, its operation was very sensitive to changing background conditions during luminosity running as well as to picking up coherent noise in the front-end electronics. 
From the trigger rates achieved during the HERA I phase, it was evident that for the HERA II phase, with increased luminosity and background, it would no longer be possible to trigger on the same low thresholds with the existing system. To achieve a significant reduction of the thresholds and still maintain stable operation in the presence of increased background, a finer granularity of the input signals, a fast topological jet algorithm as well as the need for further topological input from other trigger subsystems deemed necessary. The Jet Trigger has been designed to implement these ideas in order to maintain, or even improve, the possibility of low energy triggering.

\section{CONCEPT OF THE JET TRIGGER}

The main idea of the Jet Trigger is to identify localized energy depositions of electrons, photons and hadron jets in the LAr calorimeter, and to explore their topological correlations for a selective trigger decision. To achieve this in a very short time (less than $1 \mu \mathrm{s}$ ) at the first trigger level, a highly parallel algorithm has to be implemented in dedicated, custom-built trigger electronics.

\section{A. The Jet Trigger Algorithm}

The Jet Trigger is based on a quite simple idea: It finds all localized energy depositions in the calorimeter, centered around regions of local energy maxima in azimuthal angle $\phi$ and pseudorapidity ${ }^{2} \eta$, within a typical cone radius of $\Delta r=\sqrt{(\Delta \eta)^{2}+(\Delta \phi)^{2}} \approx 1$. In an offline physics analysis, the algorithm to do this would typically start with the largest (transverse) energy deposition in the calorimeter and sum up the neighboring energies up to the point where $\Delta r=$ 1 is fulfilled. The algorithm would then resume with the largest remaining energy deposition, until all energies in the calorimeter have been collected into jets.

Such a sequential algorithm could not be implemented due to the severe processing time limitations at the $\mathrm{H} 1$ first level trigger, where only about $800 \mathrm{~ns}$ were available for the digital part of the calorimeter trigger.

The Jet Trigger algorithm starts from the analog signals of the fine granularity trigger towers which are available in the LAr Trigger before the analog summing to BTs. For most of the calorimeter, the angular coverage of a trigger tower is about 0.2 radians. This means that regions with $\Delta r \approx 1$ can be formed by summing only the nearest neighbor trigger towers around a local (transverse) energy maximum to define a jet. The information content of a jet is then its transverse energy as well as its position in $(\theta-\phi)$ space. As the low energy depositions mostly originate from electronic pickup noise in the calorimeter, it is evident that only an energy-ordered list of such jets leads to meaningful and fast trigger decisions. The trigger decision can then be made using the most energetic jets from the list, exploiting in addition the topological correlations between them.

\footnotetext{
${ }^{2}$ Here, $\eta$ is the pseudorapidity, defined as $\eta=-\ln \tan \frac{\theta}{2}$, where $\theta$ is the polar scattering angle.
}

\section{B. Global Layout}

In order to realize the Jet Trigger algorithm in a running experiment, a system parallel to the digital part of the LAr Trigger has been designed. The analog trigger towers coming from the LAr Trigger Big Tower Summing unit (see figure 2) are passed through five functional units of the Jet Trigger, as shown schematically in figure 3 . These units are described subsequently:

The preprocessor ADC Calculation Storage (ACS) is the first module of the Jet Trigger. Here, the analog signals of the trigger towers, separated in electromagnetic and hadronic parts, are digitized and the transverse energy is calculated using lookup tables. Finally, the digitized signals from the electromagnetic and hadronic parts are summed into so-called input towers (IT).

The Bump Finder Unit (BFU) searches for energy maxima in the $\theta-\phi$ plane, using the set of ITs, in a highly parallelized way: Simultaneously all ITs compare their own energy with the energy of their immediate neighbors. The most energetic IT among its immediate neighbors is identified as a jet center and the jet energies are determined by adding the energies of all immediate neighbors to the jet centers (see figure 4). The result of the bump finder unit is a list of jets defined by their transverse energy $E_{T}$ and position $(\theta, \phi)$.

The Primary and Secondary Sorting Units (PSU, SSU) are two modules for a two-step sorting of the jets by decreasing transverse energy. In the first step the PSU sorts the jets within a quadrant of the calorimeter and passes on the six highest-energy jets per quadrant for further processing. In the second step the SSU combines the pre-sorted jet lists from the four quadrants and sorts the combined lists in decreasing transverse energy. The final result of the SSU is a list of the 16 highest-energy jets, sorted in decreasing order, the so-called jet-container. The jet-container collects the information about the transverse energy and position of each of these jets.

The Trigger Element Generator (TEG) houses the necessary logic to prepare the trigger decision from the energysorted list of jets. Typical actions of the TEG are to discriminate the individual jet energies, to count jets with energies above programmable thresholds and to determine topological correlations on the basis of the angular information of the jets. The TEG is designed in a very flexible and expandable way to be able to follow changing physics interests and optimal triggering strategies.

\section{REAlization OF THE JET TRigger}

The Jet Trigger algorithm, though simple in principle, necessitates a rather demanding realization as it has to be fully executed in 8 bunch crossings, i.e. a little less than $800 \mathrm{~ns}$. The latency requirements of the Jet Trigger units are as follows: The 1200 analog trigger tower signals (about $1 \mu \mathrm{s}$ in width) are sampled at the HERA clock frequency, corresponding to the time unit of one bunch crossing (BC). If one considers, for example, the sample $\mathrm{N}$ at bunch crossing 0 , relative to the delivery of the analog signals to the ACS FADC system, the digital value is obtained at $\mathrm{BC} 1$. The further summing and storing steps in the ACS unit take another 2 BCs. At BC 3 the 


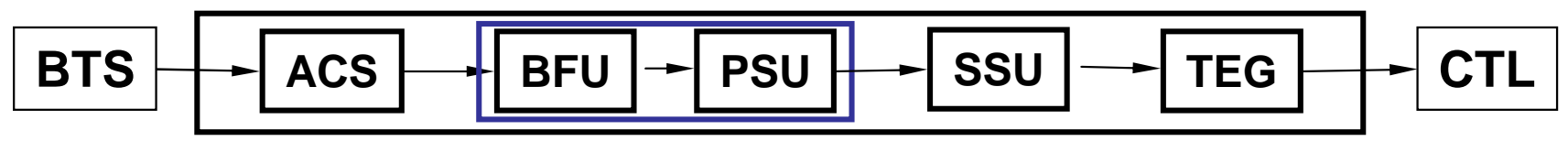

Fig. 3. Schematic layout of the functional units of the Jet Trigger.

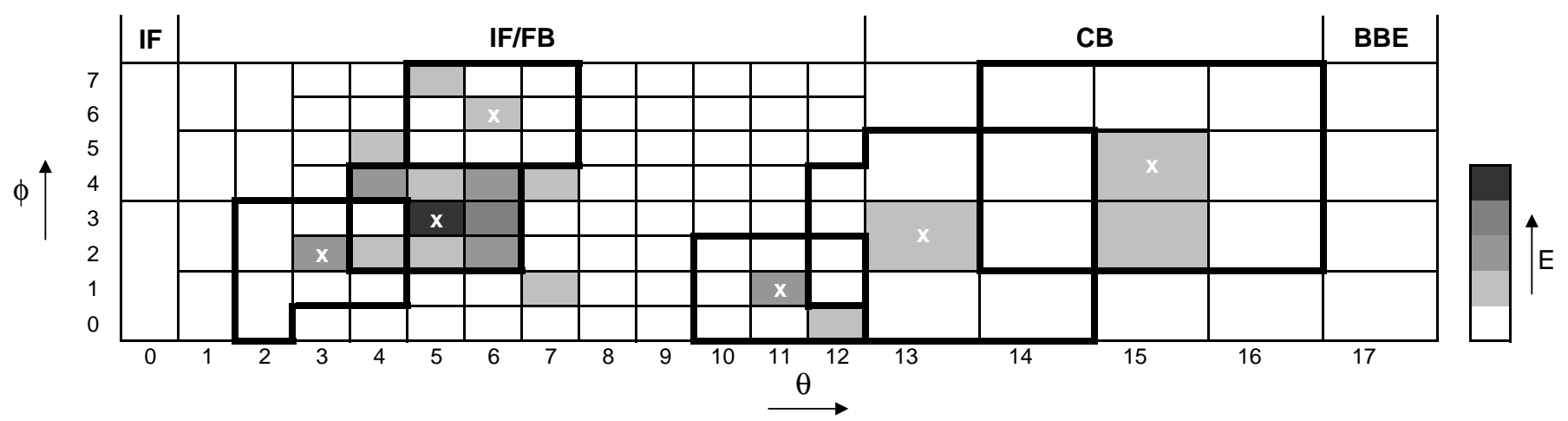

Fig. 4. Schematic overview in $\theta$ and $\phi$ of input towers in one quadrant. The $\theta$ index 0 (17) corresponds to the most forward (backward) region in the calorimeter. The calorimeter is made of the inner forward and forward barrels (IF/FB), the central barrel (CB) and the backward barrel (BBE). The different shades show different energy content of the input towers. The thick lines show the borders of the jets found by the algorithm, with the jet centers marked with "x".

digital data of the pre-processed ITs are sent, over a bit-serial link, to the BFU. At BC 4 the IT data are received by the BFU. At BC 5 the output of the BFU is available, and at BC 6 the output of the PSU. At BC 7 the selected and sorted maxima are sent to the TEG, where the final trigger elements are generated at BC 8. All these operations are implemented in a highly interconnected array of FPGAs. In total the Jet Trigger consists of 550 FPGAs with 75 million gates, computing 300 giga operations per second. The $12 \mathrm{~GB} / \mathrm{s}$ raw data rate is reduced to 16 trigger element bits per bunch crossing, corresponding to a data reduction factor of 600 .

\section{A. New Big Tower Summing Unit}

The analog signals for the Jet Trigger originate from a new Big Tower Summing unit (BTSN), replacing the corresponding unit of the LAr trigger (see section II-A). This module has to provide the analog big tower signals to the LAr Trigger and, in addition, the higher granularity trigger tower signals to the Jet Trigger.

As can be seen in figure 5, the BTSN receives groups of 64 analog trigger towers, i.e. 32 EM and 32 HAD channels. The input signals branch into two different lines. One of them sums up to 4 adjacent towers into a big tower using an analog summing unit, amplifies the sums and outputs them on coaxial cables to the FADC boards of the LAr Trigger. This functionality already existed in the former version of the BTS. The analog summing and amplification in the BTSN is done with a wideband, low noise operational amplifier.

The BTSN has in addition a separate line which forwards each of the 64 raw trigger towers directly to the ACS. As the cables between BTSN and ACS are about $10 \mathrm{~m}$ long, an analog differential signal transmission with shielded twisted pair cables is used. This gives good independence to ground and very little susceptibility to noise pickup. Figure 6 shows a picture of the BTSN printed-circuit board (PCB).

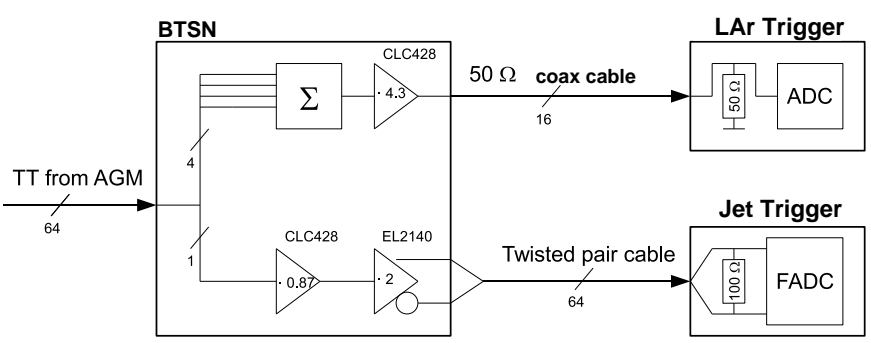

Fig. 5. Schematic layout of the new Big Tower Summing unit (BTSN) with two branches: The big tower sums on top go to the LAr Trigger, the individual trigger towers on bottom go to the Jet Trigger.

The analog signal level of a TT is adjusted with an operational amplifier in such a way that the energy seen in a TT gives the same number of ADC counts in the LAr Trigger and the Jet Trigger. The LAr Trigger ADC has a stroke of $6 \mathrm{mV}$ per ADC count. Each analog trigger tower signal is amplified by a factor of 4.3. The Jet Trigger ACS has a stroke of $2.44 \mathrm{mV}$ per ADC count. The signal to the ACS is amplified by a factor of 0.87 by a low noise amplifier, and by a factor of 2 by the output driver.

\section{B. Clock Distribution and Configuration Card}

The communication between the Jet Trigger and the H1 trigger control is done via the Clock Distribution and Configuration Card (CDCC). The CDCC is connected to the Subdetector Trigger Control unit (STC) of the H1 experiment. It receives the control signals L1Keep, L2Keep, L3Keep and L2reject from the STC when an event is accepted or rejected at a given trigger level (see figure 1). The signals are processed in an FPGA within the CDCC and distributed to the Jet Trigger modules to initiate and to control their readout. The CDCC also sends information in the opposite direction, for example the Front-End-Ready (FER) signal from the Jet Trigger to 


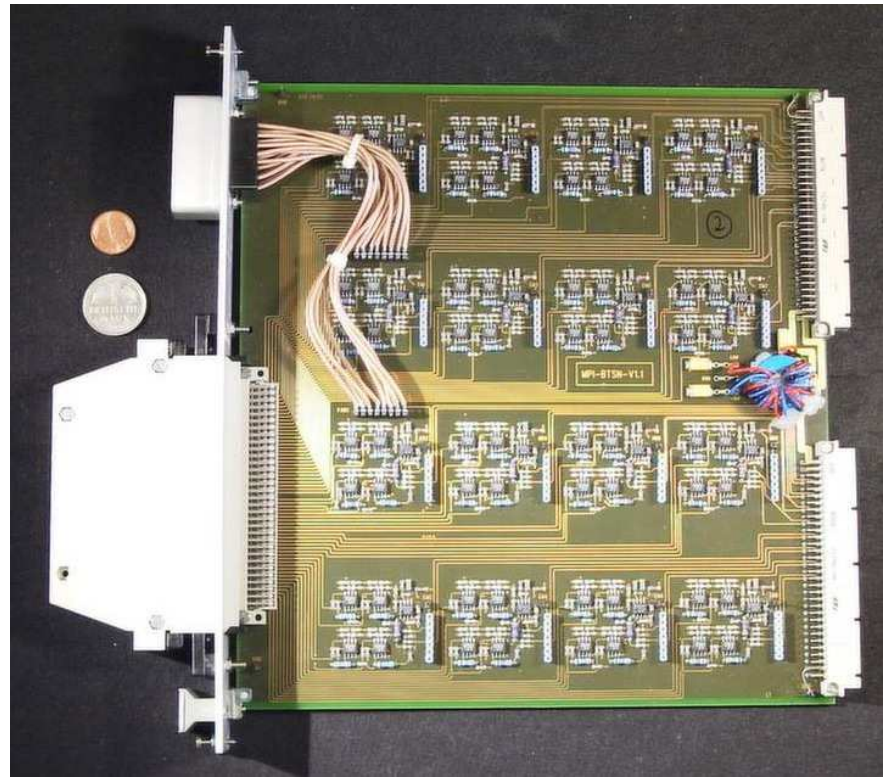

Fig. 6. Picture of the BTSN PCB with attached connectors for the differential cables to the Jet Trigger (bottom left). On top the connector for the coaxial cables providing big towers to the LAr Trigger can be seen.

the STC, indicating that the system is ready for a new event readout.

The CDCC receives the HERA clock signal. From this $10 \mathrm{MHz}$ clock it generates a $50 \mathrm{MHz}$ clock using a phase locked loop (PLL). The CDCC distributes both clock signals to the Jet Trigger modules with adjustable phases to minimize the overall system latency. The HERA clock control is implemented in such a way that it is possible to steer and readout the CDCC even when the HERA clock signal is not available or has an error. The CDCC carries 22 daughterboards to perform the clock shifts and adjustments, and 20 transceiver cards to send and receive the different signal standards of the H1 experiment.

In addition to the clock distribution, the CDCC also steers the ACS configuration. Configuration files are loaded over VME into big RAMs inside the CDCC FPGA. When the CDCC receives a configuration command, an internal state machine starts to configure all 440 ACS daughterboard FPGAs and the 56 ACS motherboard FPGAs. When the configuration is done, the CDCC collects the conditions of all 496 FPGAs in the ACS system over a serial bus and declares the system ready for taking data.

\section{Preprocessor ADC Calculation Storage}

The analog pulses from the electromagnetic and hadronic trigger towers are presummed to establish the required input tower granularity, then digitized and passed through lookup tables (LUT) where energy thresholds and calibration weights are applied. Finally, the two digitized transverse energies (EM and HAD) are summed to provide the input towers (IT). The entire algorithm is executed in a total of $300 \mathrm{~ns}$ with an additional $100 \mathrm{~ns}$ for the transmission of the ITs to the Bump Finder Unit.

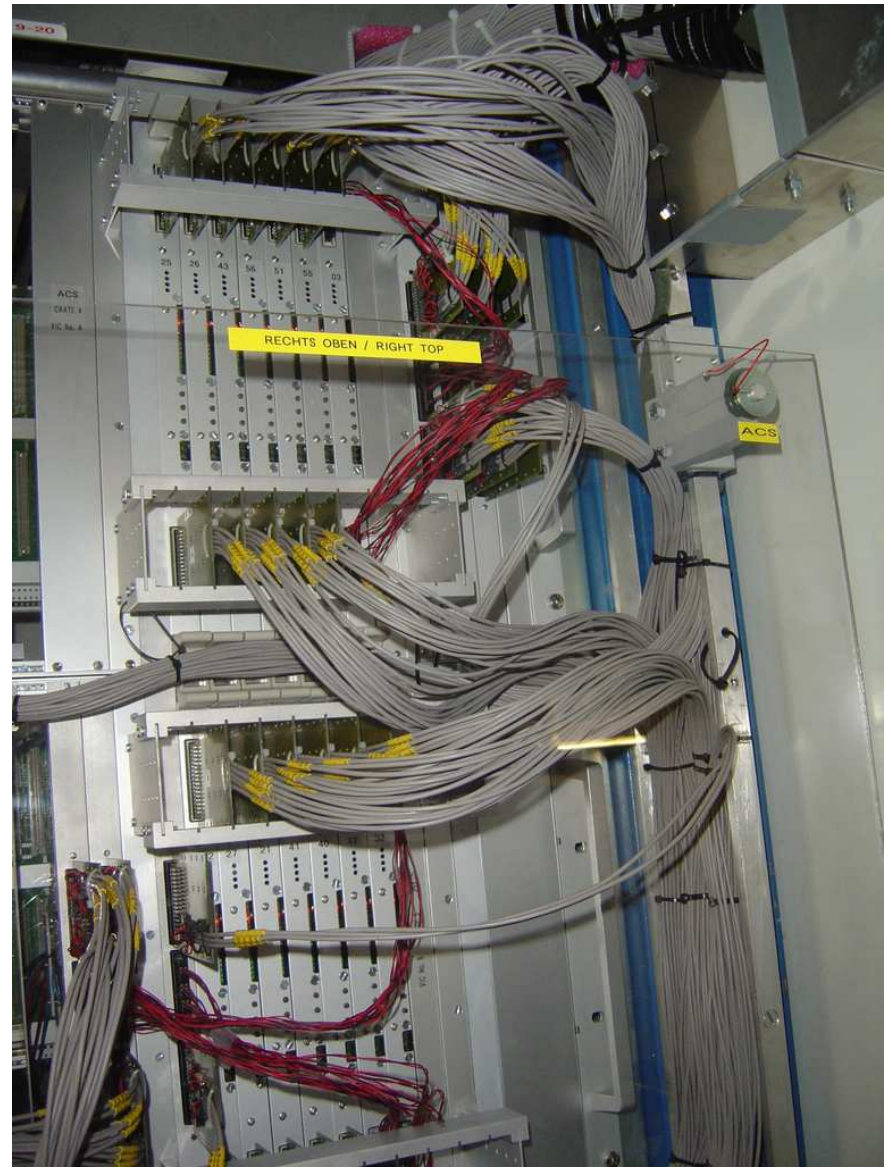

Fig. 7. Details of the installed hardware in the electronic trailer of the $\mathrm{H} 1$ experiment: View of a quarter of the Jet Trigger ADC Calculation Storage unit (ACS) which was composed of 8 crates, one for each of the 8 octants of the LAr calorimeter. The ACS receives the analog signals of the projective trigger towers and transfers the digitized signals to the Bump Finder Unit (BFU) via a bit-serial link.

The ACS is the largest system of the Jet Trigger and consists of 24 summing boards, 56 motherboards, each equipped with 8 daughterboards, and one CDCC. The full system is mounted in eight 9U VME crates, each of them serving one octant of the calorimeter. Part of the system, as it was mounted in the H1 experiment, is shown in figure 7.

An overview of the signal processing in the ACS is shown in figure 8 . The system receives the 568 analog values of the electromagnetic trigger towers and the 624 analog values of the hadronic trigger towers from the BTSN over 1192 cables, each $10 \mathrm{~m}$ long.

The trigger tower signals have different granularities depending on their $\theta$ positions (see figure 4). The signals with finer granularity are first sent to the summing boards where they are summed to the desired input tower granularity which is optimized as a function of $\theta$ in order to approximate a cone radius $\Delta r \approx 1$ for all pseudorapidities. The signals coming from the forward region already have the needed granularity and do not pass the summing step.

The analog signals are then sent to the ACS motherboards and further distributed to daughterboards (see figure 9). On the daughterboards, the signals are first digitized by a set of 10-bit ADCs. Because of the long pipeline latency of the ADC 
ACS system

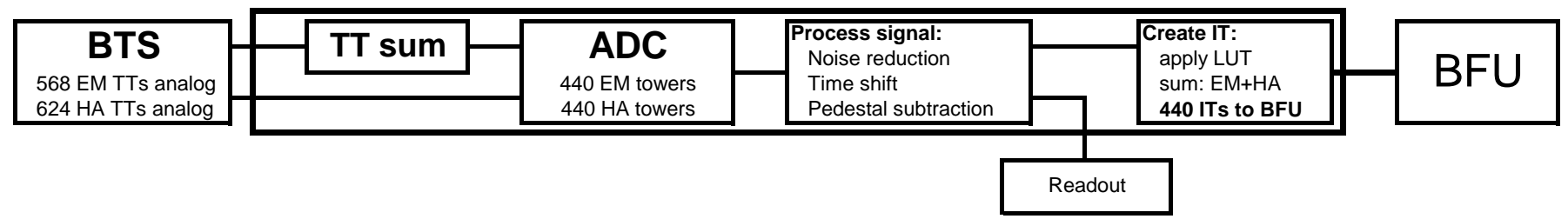

Fig. 8. Schematic overview of the various steps of the ACS signal processing (see text for details).

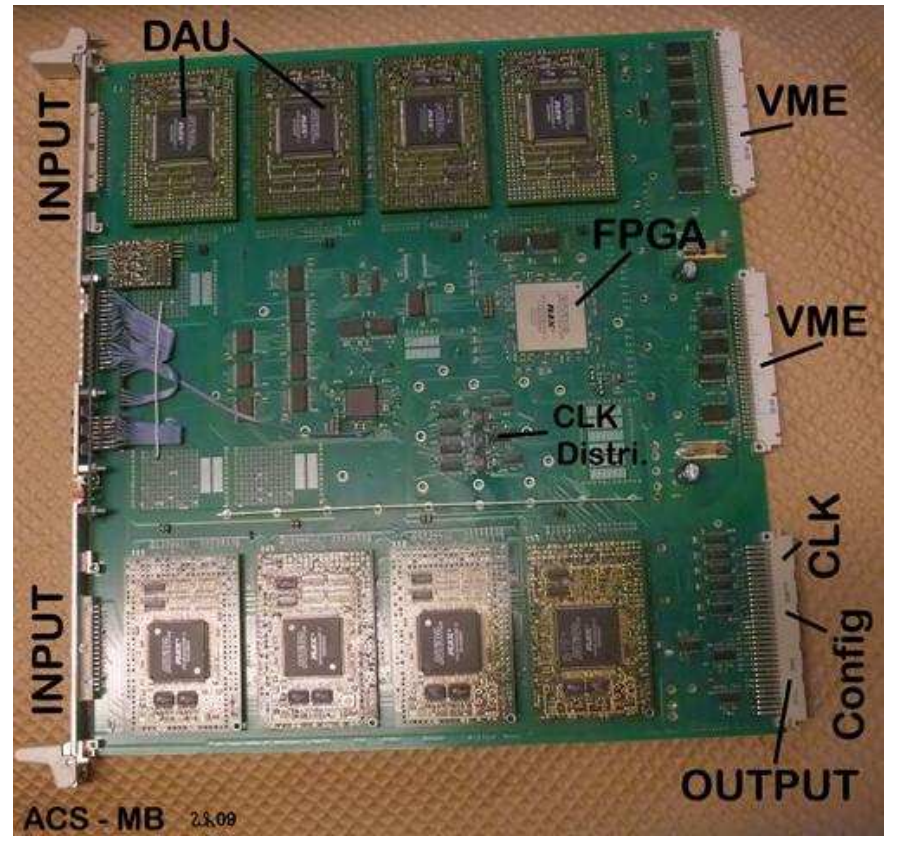

Fig. 9. Picture of the ACS motherboard with 8 daughterboards (DAU).

the sample clock is $50 \mathrm{MHz}, 5$ times the HERA clock. In this way the samples are available after $100 \mathrm{~ns}$. In order to reduce the noise contribution in the digital signal, only the higher 9 bits are used.

The analog signals from the BTSN do not all arrive at the ACS at the same time, caused by the different cell capacities of the towers in the LAr calorimeter: The signals from the forward part of the detector (small cell capacities) arrive first and the signals from the central and backward part of the detector (large cell capacities) arrive last. The time difference between the slowest and the fastest signals is about $100 \mathrm{~ns}$. In order to synchronize all the signals, the faster signals are delayed by 100 or $50 \mathrm{~ns}$ depending on the cell capacities.

The next step of the signal processing is the offset and pedestal subtraction with parameters for each individual signal, programmable over VME. The highest bit is then no longer used. At this point the data stream is separated in two independent paths.

One path prepares the signals for the readout. A state machine in the daughterboard FPGA is designed to retrieve the stored values from the input pipeline and to shift them into registers. These registers are then read from the motherboard collection state machine and are prepared for the calorimeter readout. The FADC values are zero-suppressed with programmable thresholds. A double buffering mechanism is implemented to avoid creating second-order deadtime in case of readout requests arriving before the current readout is completed.

On the second path, the data processing continues on the daughterboards. Here, an optimized $\theta$-dependent noise cut is applied and the transverse energy is calculated, using geometry-dependent factors. This is done by applying $256 \times$ 8 bit LUTs, which are read and written via VME. Then the electromagnetic and hadronic signals on each daughterboard are summed into an input tower and sent to the BFU. The signal processing requires $200 \mathrm{~ns}$.

Finally, the daughterboard FPGAs are responsible for storing the data stream in the output pipeline for later readout. The pipeline can also be loaded over VME with test patterns in order to debug and optimize the transmission delay of $100 \mathrm{~ns}$ to the BFU. The resulting 440 input towers are transferred via a bit-serial link to the BFU.

\section{Bump Finder Unit}

The highly networked Bump Finder Unit where each IT is digitally connected to each of its up to 10 neighbors receives the digitized values from the ACS and searches for local maxima of energy, the so-called "bumps". This search is done for each IT in a completely parallel fashion. The energy content in each input tower is compared with each of its nearest neighbors (see figure 4). If an IT has found that all of its neighbors have less energy than the IT itself, the IT is declared to be a jet center and the energies of all its neighbors are added to the energy of the IT. If two ITs have the same energy (same energy and same $\theta$ position) the one with the larger $\theta$ (larger $\phi$ ) is declared to be the jet center. The evaluation of jet centers and the formation of the jet energies are performed in FPGAs within 100 ns.

The BFU which is divided into two motherboards, one for each hemisphere, is shown in figure 10. Each of them maps two identical quadrants. The two PCBs (see figure 11) are located in a VME crate face-to-face. Using such a positioning of the motherboards, only short cables are used for the neighbor connections. The connections with the ACS signals are placed on the outer side of the boards.

The Bump Finder network connects all ITs with their immediate neighbors, reflecting the barrel structure of the calorimeter. The input towers are partitioned in four quadrants. Each quadrant contains 110 input towers and is organized in 29 groups containing 2 or 4 input towers. Only one input tower in such a group can be a jet center. Two neighboring jets are 


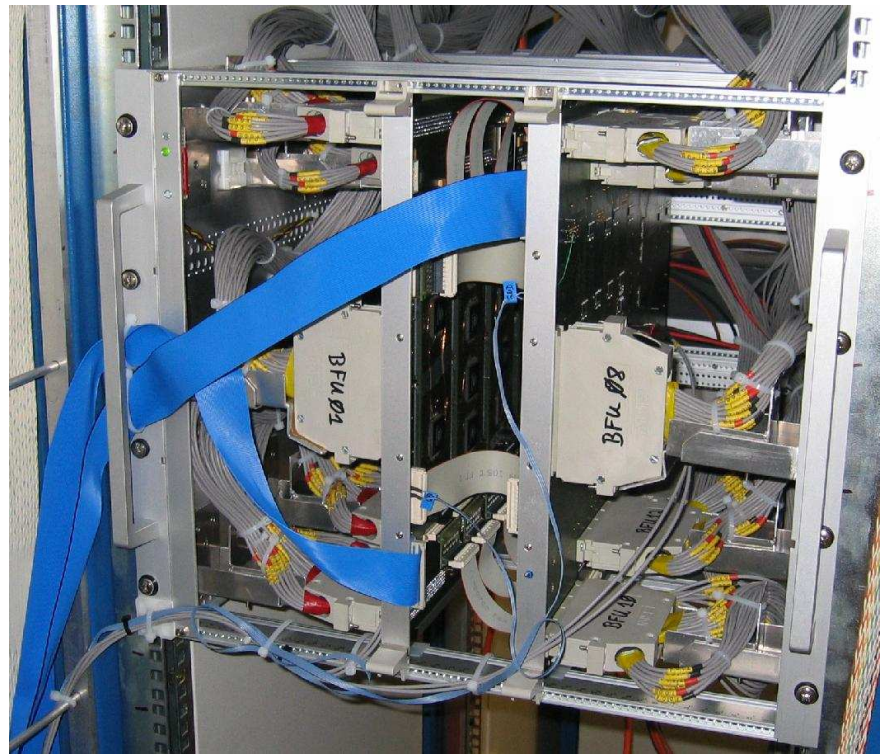

Fig. 10. View into the Bump Finder crate containing 2 Bump Finder Units (BFU), one for each calorimeter hemisphere.

separated by at least one input tower, i.e. a saddle point must exist. This results in a maximum of 29 jets per quadrant. The jet energy ( 8 bits) and the address within a group ( 2 bits) are transferred to the Primary Sorting Unit. The group number is known by its wiring and does not need to be transferred.

\section{E. Primary Sorting Unit}

The jets found by the BFU are sorted by decreasing transverse energy. This is done in two steps, first for each quadrant separately by four Primary Sorting Units (PSU). Each PSU receives 29 jets per quadrant (most of them will have zero energy) and outputs the six highest-energy jets, sorted by energy, to the Secondary Sorting Unit. The remaining 23 jets are dropped. This cut removes jets with zero energy and part of the noise. If two jets have the same energy, they are sorted by $\theta$ and $\phi$ in the same way as in the BFU. The algorithm of the energy-sorting in the PSU is implemented in FPGAs located on the BFU motherboards (see figure 11). The latency of the PSU is one bunch crossing.

Each PSU receives 29 jets from the BFU, the jet information consisting of an 8-bit energy and a 2-bit local address. In a first step the local address together with the jet group number, which is known by the wiring, are transformed into a nonambiguous input tower address. For the 110 input towers per quadrant a 7-bit value is needed to cover the full range. The address calculation is done by a predefined lookup table in such a way that a higher address is related to a higher priority in $\theta$ and $\phi$. The energy value is then extended by the calculated address to the right, giving a 15-bit value for each jet:

$$
\underbrace{b_{14} \ldots b_{7}}_{\text {energy }} \underbrace{b_{6} \ldots b_{0}}_{\text {address }}
$$

This simplifies the comparison procedure also in the case of equal energy in two jets.

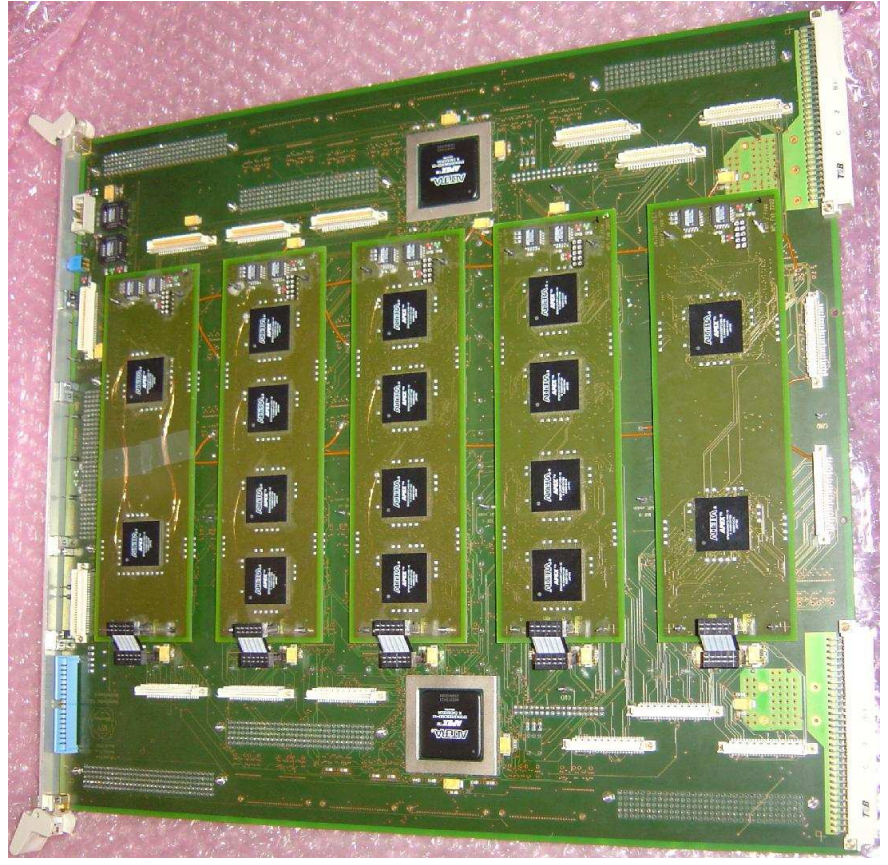

Fig. 11. BFU motherboard, equipped with daughterboards. The white 50pin connectors are the neighbor connections to the second board. The blue connector on the left connects to the VME controller board. The small FPGAs implement the BFU algorithm, the two large ones the PSU algorithm.

The sorting algorithm is carried out in a parallel manner, similar to the jet finding algorithm. Each jet compares its energy and its address with all the other jets. If jet $t_{\mathrm{a}}>$ jet $_{\mathrm{b}}$, the comparison bit for $\mathrm{a}-\mathrm{b}$ is set to 1 . In order to avoid double comparison the bit for $b-a$ is set to 0 at the same time. Each jet determines the number of jets with smaller or equal energy by incrementing a counter which represents the sum of the comparison bits. The jet with sum 28 is the largest (all the other 28 jets have smaller energy), the one with sum 27 is the next, and the one with sum 0 is the lowest. The first six jets (counter number 28..23) are sent to the output registers and transmitted to the SSU.

\section{F. Secondary Sorting Unit}

The 24 jets from the PSU, sorted quadrant-wise by decreasing transverse energy, are transferred to the Secondary Sorting Unit where they are sorted again to obtain the energy-sorted list of jets found in the entire calorimeter. The list of the 16 largest jets is sent to the Trigger Element Generator.

Physically, the SSU is a modified version of the TEG motherboard and can be seen in figure 12. The SSU algorithm is implemented in FPGAs with a latency of one bunch crossing.

The sorting algorithm in the SSU is rather similar to the PSU algorithm. First the 10-bit jet address ( 5 bits for $\theta, 5$ bits for $\phi)$ is built from the 7-bit PSU address in conjunction with the quadrant information which is known by the wiring. Adding the address to the energy gives a full value of 18 bits:

$$
\underbrace{b_{17} \ldots b_{10}}_{\text {energy }} \underbrace{b_{9} \ldots b_{5}}_{\theta} \underbrace{b_{4} \ldots b_{0}}_{\phi}
$$


In this way the jets are sorted by energy, $\theta$ and $\phi$, respectively, following the same logics as in the BFU and SSU: Each jet is compared with all the other jets. If jet $\mathrm{a}_{\mathrm{a}}>$ jet $_{\mathrm{b}}$, the comparison bit $\mathrm{a}-\mathrm{b}$ is set to 0 and the comparison bit $\mathrm{b}-\mathrm{a}$ to 1. All comparison bits of each jet are summed up. The jet with sum 0 has the highest energy, the one with sum 1 the second highest energy and so on until the least energy jet with sum 23. Finally, a list of the 16 highest-energy jets, sorted by decreasing energy, is produced. This cut removes part of the remaining noise in the energy depositions.

The number of 16 jets has been chosen from a study of deep inelastic scattering events to not lose significant energy depositions. This list of 16 jets, ordered in transverse energy, is referred to as the jet-container. The jet-container is sent to the Trigger Element Generator to perform the first level trigger decisions. In addition, the jet-container is sent to the second level trigger where it is correlated with the information from other trigger subsystems, for example the FTT.

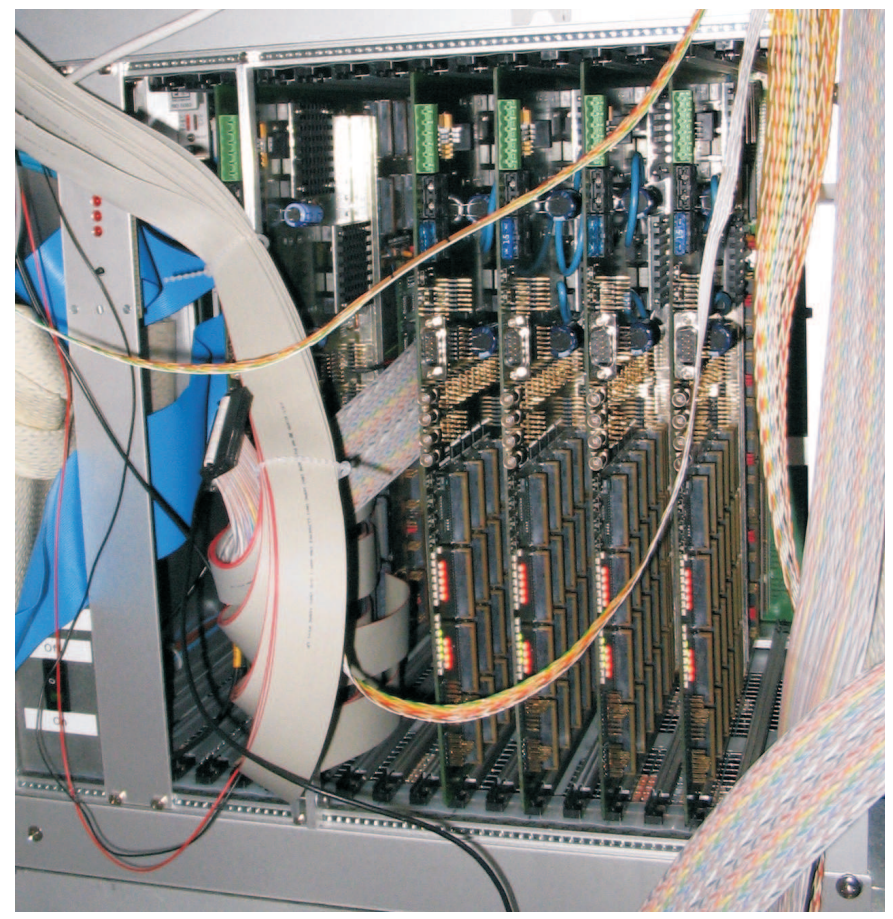

Fig. 12. View into the crate housing the Secondary Sorting Unit (SSU, the left most board) and the 4 Trigger Element Generator units (TEG).

\section{G. Trigger Element Generator}

The trigger elements are the basic logical input to the CTL, where the first level trigger information from all the subdetectors is collected and combined to subtriggers. The programmable TEG (see figure 12) applies conditions on the energy and location of the jets from the jet-container to generate the trigger elements leading to an actual trigger decision signal L1keep.

The jet-container is realized as an ordered bus on the backplane of the TEG crate. The information transferred per jet is its transverse energy, $\theta$ and $\phi$. Since the jet-container is a bus, an arbitrary number of units may have access to it and a trigger element can be easily added if needed without disturbing the determination of the trigger elements already implemented in the Jet Trigger.

The TEG is implemented on a motherboard which was designed to also be used for the SSU. To reduce the number of necessary TEG boards and the number of active receivers on the jet-container bus, 4 TEG units are realized on one board. Each board produces 4 trigger elements. The TEG system is built from 4 separate, identical boards, which can prepare up to 16 trigger elements. The TEG data stream processing is separated into two different parts: The "global" TEG and the "local" TEG which are executed in parallel on separate FPGAs. The full TEG logic is executed within one bunch crossing.

The TEG algorithm constructs trigger elements from subelements. The sub-elements are classified as local trigger elements produced by the local TEG, and global trigger elements produced by the global TEG. Local trigger elements are calculated by applying conditions on individual jets, while global trigger elements are calculated by performing sums on the jets in the jet-container. A trigger element is the result of a logical expression combining a global trigger element and a local trigger element. All logical operations are possible: logical AND, logical OR, negation and expressions with brackets.

The global TEG determines 4 global trigger elements for the corresponding 4 local TEG units. A global trigger element is formed from a logical operation combining up to eight global trigger conditions (GTC) which are similar to the global sums $E_{T}$ and $E_{T}^{\text {miss }}$ of the LAr Trigger. The global TEG loops over the jet-container and computes the jet energy components $E_{x}$ and $E_{y}$ using a lookup table for the sin and cos functions of the angles. Then it calculates the global sums from jets fulfilling preloaded conditions on positions in the jet-container or $\theta$. The energies of the selected jets are summed up by a generic adder tree to compute $E_{T}$ and $E_{T}^{\text {miss }}$. These sums are then compared with preloaded energy thresholds. There are up to 4 GTCs based on $E_{T}$ and up to 4 GTCs based on $E_{T}^{m i s s}$. Contrary to the LAr Trigger, $E_{T}$ and $E_{T}^{\text {miss }}$ can therefore be calculated in programmable restricted $\theta$ ranges, for instance only in a given region of the calorimeter, or to avoid a noisy region. $E_{T}$ and $E_{T}^{m i s s}$ can also be calculated from a subset of jets in the jet-container, for instance from the first four leading jets. The logic equation which forms the final global trigger element is programmable and allows any logical combination of the precalculated global trigger conditions for any of the 4 TEG units on a board.

There are 4 local TEG units per TEG board giving 4 local trigger elements to be combined with the global trigger elements. Each local trigger element is composed of a logical combination of up to eight local trigger conditions (LTC). The local TEG first selects the leading jet from the jet-container, which is the first jet in the list fulfilling freely selectable conditions on its position in the list, its energy and polar angle $\theta$. Then the energy difference $\Delta E$, the polar and azimuthal angular differences $\Delta \theta$ and $\Delta \phi$ of the other jets relative to the leading jet are calculated, all fully in parallel. As an example, 
the following conditions can be implemented between 2 jets:

$$
\begin{aligned}
& E_{\text {cut } 1, l} \leq E_{1} \leq E_{\text {cut } 1, h} \quad \text { and } \quad \theta_{\text {cut } 1, l} \leq \theta_{1} \leq \theta_{\text {cut } 1, h}, \\
& E_{\text {cut } 2, l} \leq E_{2} \leq E_{\text {cut } 2, h} \quad \text { and } \quad \theta_{\text {cut } 2, l} \leq \theta_{2} \leq \theta_{\text {cut } 2, h}, \\
& \Delta E_{\text {cut }, l} \leq \Delta E \leq \Delta E_{\text {cut }, h} \quad \text { and } \quad \Delta \theta_{\text {cut }, l} \leq \Delta \theta \leq \Delta \theta_{\text {cut }, h}, \\
& \Delta \phi_{\text {cut }, l} \leq \Delta \phi \leq \Delta \phi_{\text {cut }, h} .
\end{aligned}
$$

These conditions form the local trigger element bit which is then used together with the global trigger element bit to form a final trigger element sent to the H1 Central Trigger Logic. For test purposes the data processing pipeline of the TEG unit, like all the other Jet Trigger units, can be preloaded with arbitrary test patterns, producing test results at full speed and in real-time.

\section{H. Data Acquisition and Readout}

The readout of the Jet Trigger was integrated directly into the existing $\mathrm{H} 1$ readout [23]. This had a number of advantages. First, the design of new data acquisition hardware was avoided. Furthermore, the additional online programming for the data acquisition was minimized. Finally, this ensured that the new level 1 trigger quantities determined by the Jet Trigger reached the higher level systems in the standard way and minimized the necessary adaptations. These goals were achieved by the integration of a double buffer system in the ACS, thus avoiding the use of DSPs for the readout.

Each Jet Trigger module, with exception of the PSU, had the possibility to send both the input and the output data to the readout, allowing for a full monitoring and control of the functionalities of all Jet Trigger components. The full readout created a data volume which exceeded the readout limits during standard luminosity running. Therefore, the Jet Trigger readout had two modes: The standard readout used during luminosity data taking which was sufficient to check the digital consistency of the trigger, and the full readout used for testing, debugging and control of the Jet Trigger.

In standard readout, the electromagnetic and hadronic FADCs from the ACS system were read out in a zerosuppressed way. Within $10 \mu$ s the ACS compared the FADCs counts to programmable energy thresholds and flagged those FADCs to be read out. Until the readout had been performed by a dedicated LynxOS board over VME, the ACS buffers were protected against overwriting, which was equivalent to a buffer depth of two, before creating second-order deadtime. In addition, the jet-container from the SSU and the trigger elements from the TEG unit were read out for each event without any intermediate buffer in less than $20 \mu$ s, thus impacting the existing buffer-free calorimeter readout in a minimal way.

The full readout provided the most detailed information possible. In addition to the standard readout, the FADCs below thresholds from the ACS could be read out, as well as the input towers at the BFU input, the list of 116 jets found by the BFU and the energy presorted list of jets at the SSU input.

\section{Simulation}

The Jet Trigger simulation has been developed and integrated in the H1 trigger software for an offline cross check of its performance. Depending on the purpose of the simulation, the readout data or the trigger tower simulation were used as input data. A bit-precise simulation of the hardware was performed following closely the modularity of the Jet Trigger itself. Each Jet Trigger module was simulated separately and could be run independently of the others. These features allowed for a direct, bit-precise comparison of the hardwareprocessed signals and the simulated ones. The simulation was extensively used during the testing and commissioning phase of the Jet Trigger installation. Later, the simulation was calibrated and has been used for physics measurements [24].

\section{J. Higher Trigger Levels}

The Jet Trigger provided the list of energy-sorted jets also to higher level triggers. The integrity of the Jet Trigger readout was checked by comparing online the information read out at the first and second level triggers. The list of jets was sent to the second level Neural Network trigger [18] for online monitoring of the jet distributions. The jets were also sent to the third level Fast Track Trigger [25] to combine them with tracks measured in the central jet chambers and to perform electron identification.

\section{Jet Trigger Performance}

The entire hardware of the Jet Trigger electronics was designed, built and successfully tested at the Max-PlanckInstitut für Physik in Munich and was fully installed in the electronic trailer of the $\mathrm{H} 1$ experiment in the year 2005. With the Jet Trigger hardware fully installed, the commissioning phase of the trigger began in the fall of 2005. After an intense period of debugging this highly complex system, the operation of the Jet Trigger started in the summer of 2006.

The energy-sorted jet information was combined with higher level track-based triggers to successfully perform electron identification with a momentum threshold as low as $1.2 \mathrm{GeV}$. In the fall of 2006, the Jet Trigger was fully integrated into the $\mathrm{H} 1$ trigger system, also providing triggers at the first trigger level. This enlarged the phase space for events containing a single forward jet with polar angles below $30^{\circ}$. In spring of 2007, the energy of the HERA proton beam was decreased from $920 \mathrm{GeV}$ to $460 \mathrm{GeV}$. This allowed to measure the neutral-current inclusive cross sections at different $e p$ centerof-mass energies and opened up the possibility to directly extract the longitudinal structure function $F_{L}$ of the proton.

The contribution of $F_{L}$ to the cross section is sizable only at high inelasticity $y=1-\frac{E^{\prime}}{2 E}(1-\cos \theta)$, where $E$ and $E^{\prime}$ denote the energy of the electron before and after the interaction and $\theta$ is the polar angle of the scattered electron.

For a measurement of $F_{L}$ it is therefore mandatory to record events characterized by low energy scattered electrons which were not triggered by the LAr Trigger due to its high threshold of $6 \mathrm{GeV}$. The Jet Trigger was used to successfully decrease the electron triggering threshold from $6 \mathrm{GeV}$ down to $2 \mathrm{GeV}$ and to perform the world's first direct measurement of the 
longitudinal structure function $F_{L}$ of the proton at high $Q^{2}$. An integrated luminosity of about $100 \mathrm{pb}^{-1}$ was accumulated with the Jet Trigger active until the termination of the HERA II program by the end of June 2007.

\section{A. Jet Performance}

Triggering on events with a hadronic final state in the forward direction was not possible with the LAr Trigger due to large rates resulting from energy deposits of the proton remnant distributed all over the forward part of the calorimeter. To control these rates the thresholds in the forward region had to be set very high. For this reason the charged-current measurement suffered from insufficient trigger efficiency for events with a jet in the forward direction, i.e. with low values of the polar angle of the hadronic final state $\gamma_{\text {had }}$.

In the fall of 2006 this situation was improved by using the Jet Trigger at the first trigger level. The Jet Trigger was configured to select events which had a jet in the forward direction with a polar angle $\theta_{\text {jet }}<30^{\circ}$. A threshold in transverse energy of $8 \mathrm{GeV}$ was chosen as it produced a trigger rate of about $1 \mathrm{~Hz}$ which was affordable in the trigger budget while preserving a high efficiency.

Figure 13 shows the efficiency of triggering on jets in this phase space as a function of the polar angle of the hadronic final state $\gamma_{\text {had }}$, for both the Jet Trigger and the LAr Trigger. The figure shows that at the lowest angles the efficiency of all the components of the LAr Trigger vanishes whereas the Jet Trigger efficiency reaches a maximum of close to $100 \%$. The amount of data collected with this forward-jet trigger active corresponds to a luminosity of about $50 \mathrm{pb}^{-1}$.

\section{B. Heavy Quark Performance}

The Jet Trigger was also successfully used to perform electron identification and to trigger on electrons stemming from decays of low momentum b-quarks. To access leptonic decays of heavy quarks, conventional track-based triggers usually apply a low track momentum threshold (e.g. $1 \mathrm{GeV}$ ) and therefore typically suffer from high rates at the $\mathrm{kHz}$ level. Large prescale factors must be applied to record these events, limiting the statistical precision of measuring low momentum heavy quark processes.

In order to reduce the trigger rates and the prescale factors, a trigger was designed during the summer of 2006, combining the FTT and the Jet Trigger. The trigger combines the track measurements of the FTT with the energy-sorted calorimeter information coming from the Jet Trigger. The combination consists of both the kinematic and the geometric matching of the information coming from both devices to discriminate electrons from hadrons. The triggered electrons must have an FTT track pointing to a jet from the jet-container in an acceptance window as illustrated in figure 14. Due to its non-compensating design the H1 LAr calorimeter response is smaller for hadrons than for electrons of the same energy. Therefore a cut on the ratio of the transverse energies measured by the FTT and by the Jet Trigger permits a separation of electrons and hadrons.
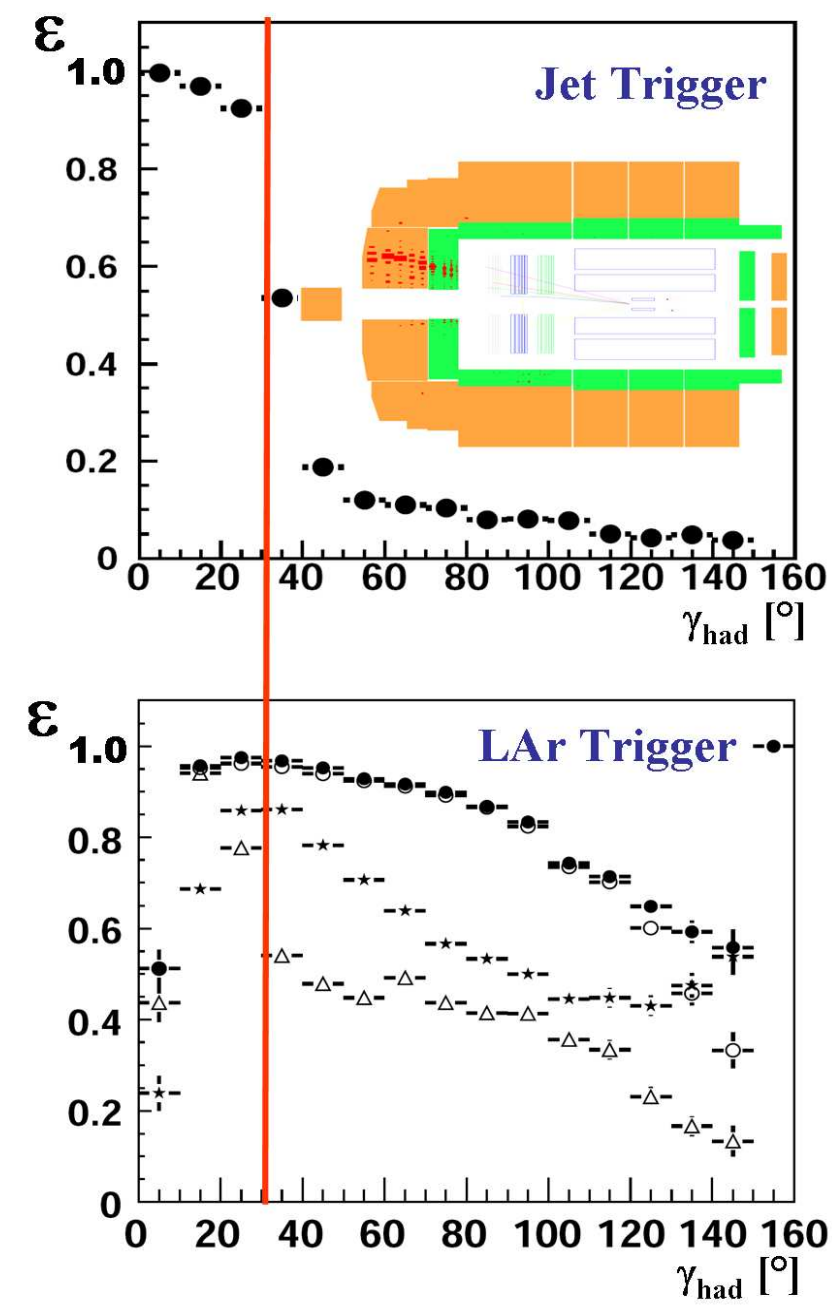

Fig. 13. Trigger efficiency for forward jets as a function of the polar angle of the hadronic final state $\gamma_{h a d}$, for both the Jet Trigger (top) and the LAr Trigger (bottom). For the LAr Trigger the efficiency of several trigger components $(\triangle, \star, \circ)$ and their combination $(\bullet)$ is given. The event display in the upper figure shows the topology of a forward jet event in the LAr calorimeter. The LAr Trigger efficiency significantly drops at angles below $30^{\circ}$, delimited by the vertical line, where the Jet Trigger efficiency reaches $90-100 \%$.

The performance of the electron trigger in data is checked with the decay $J / \Psi \rightarrow e^{+} e^{-}$. Figure 15 shows the electron identification efficiency for two single tag electron finders requiring one electron candidate with a medium $(1.5 \mathrm{GeV})$ and a high $(2 \mathrm{GeV}) p_{T}$-threshold, and for a double tag electron finder requiring two electron candidates with a low $(1.2 \mathrm{GeV})$ threshold. The rate reduction achieved ranges from a factor 15 for the medium $p_{T}$-threshold to 100 for the high $p_{T}$-threshold as shown in figure 16. Using the Jet Trigger, the rates are reduced from $100 \mathrm{~Hz}$ to $1 \mathrm{~Hz}$, acceptable for recording. Only with a double tag electron finder is it possible to implement a very low $p_{T}$-threshold of $1.2 \mathrm{GeV}$. The single tag finders reach a maximum efficiency of about $70 \%$. The higher maximum efficiency of $85 \%$ of the double tag finder is due to relaxed track-jet matching cuts. About $100 \mathrm{pb}^{-1}$ of luminosity was recorded with the electron trigger active. This large data sample is used by $\mathrm{H} 1$ to measure beauty in photoproduction 
in an extended kinematic region (see [24]).

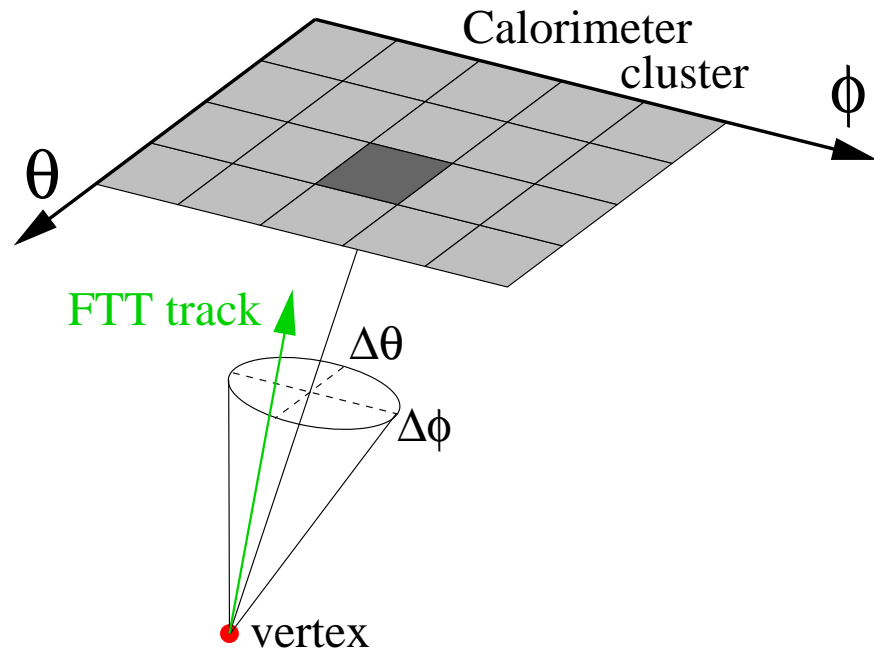

Fig. 14. The electron identification is done by allocating to every FTT track a jet from the Jet Trigger in a given acceptance window $\Delta \theta, \Delta \phi$.

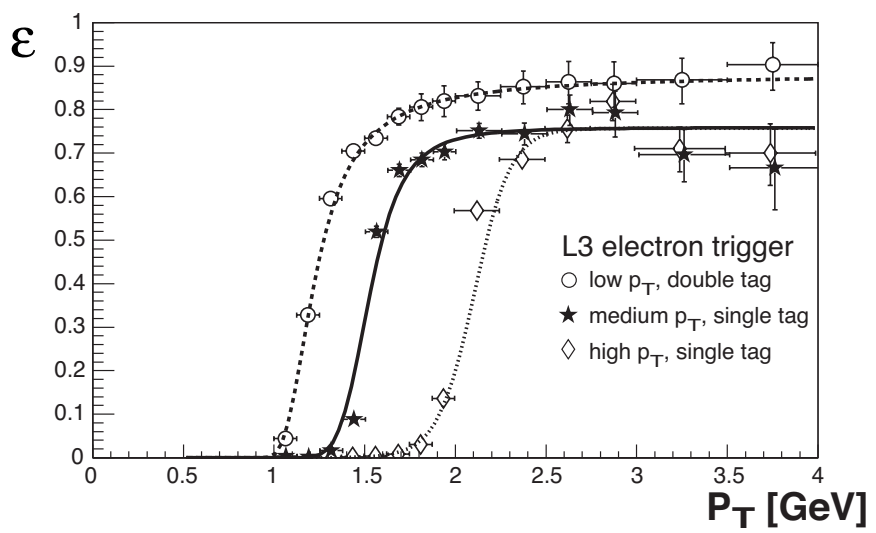

Fig. 15. The electron identification efficiency of the single tag medium and high $p_{T}$ electron finder $(\star, \diamond)$ as a function of $p_{T}$. The double tag electron finder (o) with the lowest $p_{T}$-threshold for the electron is also shown. The different $p_{T}$-thresholds of $1.2,1.5$ and $2 \mathrm{GeV}$ are clearly visible.

\section{Electron Performance}

The last three months of the HERA data taking in spring 2007 were devoted to special low energy runs with reduced proton energy. This program was launched to measure the longitudinal structure function $F_{L}$ of the proton which is dominated by the contribution from the gluon density. The LAr Trigger becomes efficient for electrons above $6 \mathrm{GeV}$ which only allows limited access to the phase space where the $F_{L}$ contribution is sizeable.

To trigger on lower energy electrons, down to $2 \mathrm{GeV}$, two triggers were combined to achieve a higher efficiency: The previously described electron trigger where a track from the first level FTT is validated at the third level with a cluster from the Jet Trigger, and a new trigger where a cluster from the first level Jet Trigger is validated at the third level with a track from the FTT. To reduce the rates of the latter first level trigger, the TEG used an optimized theta dependent threshold

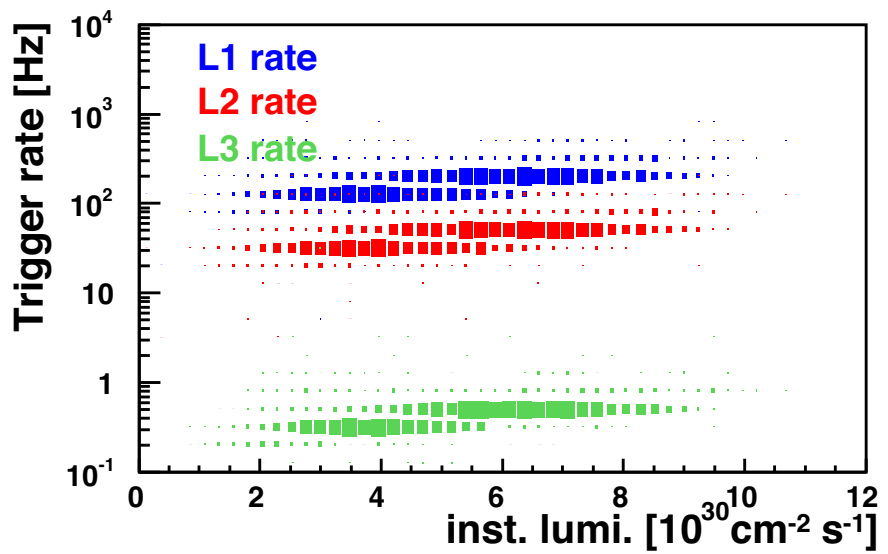

Fig. 16. Rates of the electron trigger with a $2 \mathrm{GeV} p_{T}$-threshold at the first, second and third trigger level as a function of the instantaneous luminosity. Using the Jet Trigger at the third trigger level, the achieved rate reduction factor is about 100 . The rates were reduced to $1 \mathrm{~Hz}$, acceptable for recording.

profile with the lowest energy threshold in the backward region and higher thresholds for more central electrons.

For an electron energy starting at $2 \mathrm{GeV}$, each individual trigger reached an efficiency of about $70 \%$, opening up the necessary phase space for a precise $F_{L}$ measurement. Figure 17 represents the efficiency to trigger on electrons as a function of the electron energy for both the LAr Trigger and the Jet Trigger. The threshold decrease, made possible by the Jet Trigger, is clearly visible. The $20 \mathrm{pb}^{-1}$ of luminosity collected at low proton energies with this electron trigger active have been analyzed to extract $F_{L}$ [26] and will permit to provide more accurate parton density functions of the proton.

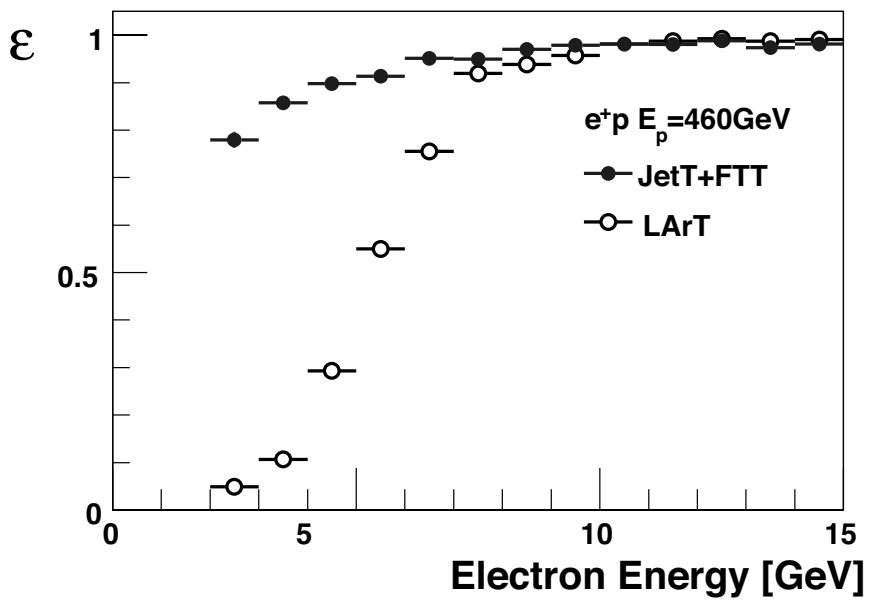

Fig. 17. Trigger efficiency for electrons as a function of the electron energy for both the LAr Trigger and the Jet Trigger.

\section{CONCLUSION}

The HERA I program was completed by the end of 2000 , with a total integrated luminosity of roughly $120 \mathrm{pb}^{-1}$ collected by the H1 experiment. The HERA II phase, which started in 2002, provided a threefold increase of the instantaneous luminosity, and was completed by the end of June 2007. The amount of data collected by the $\mathrm{H} 1$ experiment from the 
Jet Trigger activation until the end of the HERA II program corresponds to a luminosity of $100 \mathrm{pb}^{-1}$. With the Jet Trigger it was possible to substantially reduce the thresholds for triggering on electrons and jets and to record low energy events which were not kept by previous triggers. The Jet Trigger opened the phase space for events containing a single forward jet and it was used to successfully decrease the electron triggering threshold from $6 \mathrm{GeV}$ down to $2 \mathrm{GeV}$ and to perform measurements which were not possible previously.

In a future high luminosity phase of the LHC ("SLHC"), concepts similar to the Jet Trigger may be useful for the calorimeter triggers of the LHC experiments to remain sensitive to processes with low energy deposition. Also a more refined analysis of the pattern of energy deposition in the calorimeters to perform particle identification on the first trigger level, as conceptually laid out in a study of a calorimetric neural network trigger (see ref. [18]), might turn out to be extremely helpful to efficiently trigger on exotic physics.

\section{ACKNOWLEDGEMENT}

We would like to thank the German Federal Ministry of Education and Research (BMBF) for funding part of the Jet Trigger. We acknowledge the outstanding efforts of the HERA machine group and of the many technicians, engineers and physicists who worked together to construct and run the $\mathrm{H} 1$ detector over the many years of its existence. We acknowledge in particular our colleague Stefan Schmitt who, in his position as H1 trigger coordinator, was essential in the successful commissioning and running of the Jet Trigger. We also like to thank Andre Schoening who helped realizing the combination of the Fast Track Trigger and the Jet Trigger. We would finally like to honor the memory of our dear Alexey Babaev, who was our stronghold in the $\mathrm{H} 1$ control room and who could not witness anymore the success of his tireless efforts.

\section{REFERENCES}

[1] R. Hofstadter, Rev. Mod. Phys., vol. 28, pp. 214-254, 1956.

[2] R. E. Taylor, "Deep inelastic scattering: The early years," Rev. Mod. Phys., vol. 63, no. 3, pp. 573-595, Jul 1991.

[3] H1 Collaboration, DESY Report DESY-PRC-99-02, 1999.

[4] R. Maracek et al., in Proceedings of the Eighth International Conference on Calorimetry in High Energy Physics. World Scientific, October 1999, pp. 583-590.

[5] A. Aktas, Ph.D. dissertation, University of Hamburg, 2005, DESYTHESIS-2005-028,

also available at http://www-h1.desy.de/publications/theses_list.html.

[6] A. Dubak, AIP Conf. Proc., vol. 899, p. 573, 2007.

[7] B. Olivier, in proceedings of the XXVIII Physics in Collision, Perugia, Italy, June $25-28,2008$, p. 42, proceedings of the 34th International Conference on High Energy Physics, Philadelphia, USA, July 29 - August 5, 2008, proceedings of the IEEE 2008 Nuclear Science Symposium, Dresden, Germany, October 19-25, 2008.

[8] C. Kiesling, in proceedings of the 1st International Conference on Technology and Instrumentation in Particle Physics (TIPP09), Tsukuba, Japan, March 11-17, 2009.

[9] P. Schmüser, Nucl. Instrum. Meth., vol. A235, pp. 201-208, 1984.

[10] I. Abt et al., Nucl. Instrum. Meth., vol. A386, pp. 310-347, 1997.

[11] I. Abt et al., Nucl. Instrum. Meth., vol. A386, pp. 348-396, 1997.

[12] M. G. Minty, presented at the 9th European Particle Accelerator Conference (EPAC 2004), Lucerne, Switzerland, July 5-9, 2004.

[13] F. J. Willeke, prepared for 12th International Workshop on Deep Inelastic Scattering (DIS 2004), Strbske Pleso, Slovakia, April 14-18, 2004.
[14] M. Klein and R. Yoshida, Prog. Part. Nucl. Phys., vol. 61, pp. 343-393, 2008

[15] A. Baird et al., IEEE Trans. Nucl. Sci., vol. 48, pp. 1276-1285, 2001

[16] D. Meer et al., IEEE Trans. Nucl. Sci., vol. 49, pp. 357-361, 2002.

[17] J.K. Köhne et al., Nucl. Instrum. Meth., vol. A389, pp. 128-133, 1997.

[18] B. Denby et al., IEEE Trans. Neural Networks, vol. 14, pp. 1010-1027, 2003.

[19] T. Nicholls et al., IEEE Trans. Nucl. Sci., vol. 45, pp. 810-816, 1998.

[20] E. Elsen, prepared for the 2nd Annual Conference on Electronics for Future Colliders, Chestnut Ridge, USA, May 19-21, 1992.

[21] B. Reisert, Ph.D. dissertation, Ludwig-Maximilians-Universität München, 2001, MPI-PhE/2000-26, also available at $h t t p: / / w w w-h 1 . d e s y . d e / p u b l i c a t i o n s / t h e s e s$ list.html.

[22] B. Antunovic, Ph.D. dissertation, Ludwig-Maximilians-Universität, München, 2007, MPI-PhE/2007-101, also available at http://www-h1.desy.de/publications/theses_list.html.

[23] D. Hoffmann et al., Proceedings of the 10th International Conference on Calorimetry in High Energy Physics, Pasadena, USA, March 25-29, 2002.

[24] M. Sauter, Ph.D. dissertation, ETH, Zürich, Switzerland, 2009, ETH.Diss.-Nr. 18652.

[25] A. Jung et al., to be submitted to Nucl. Instrum. Meth.

[26] H1 Collaboration, in contributed paper to the XVI International Workshop on Deep-Inelastic Scattering, DIS2008, April 7-11, 2008, London,

http://www-h1.desy.de/publications/H1preliminary.short_list.html, H1prelim-08-042. 